\title{
SCD5 Expression Correlates with Prognosis and Response to Neoadjuvant Chemotherapy in Breast Cancer
}

\section{Weipeng Zhao}

Department of Breast Cancer, Tianjin Medical University Cancer Institute and Hospital, National Clinical Research Center for Cancer, Key Laboratory of Cancer Prevention and Therapy, Tianjin

\section{Linlin Sun}

Department of Breast Cancer, Tianjin Medical University Cancer Institute and Hospital, National Clinical Research Center for Cancer, Key Laboratory of Cancer Prevention and Therapy, Tianjin

\section{Xichuan Li}

Tianjin Key Laboratory of Animal and Plant Resistance, College of Life Sciences, Tianjin Normal University, Tianjin

\section{Jun Wang}

Department of Oncology, The First Affiliated Hospital of Shandong First Medical University, Jinan

\section{Ye Zhu}

Department of Breast Cancer, Tianjin Medical University Cancer Institute and Hospital, National Clinical Research Center for Cancer, Key Laboratory of Cancer Prevention and Therapy, Tianjin

\section{Yan Jia}

Department of Breast Cancer, Tianjin Medical University Cancer Institute and Hospital, National Clinical Research Center for Cancer, Key Laboratory of Cancer Prevention and Therapy, Tianjin

\section{Zhongsheng Tong ( $\nabla$ tongzhongsheng_on@163.com )}

Department of Breast Cancer, Tianjin Medical University Cancer Institute and Hospital, National Clinical Research Center for Cancer, Key Laboratory of Cancer Prevention and Therapy, Tianjin

\section{Research Article}

Keywords: Neoadjuvant chemotherapy (NACT) , breast cancer, chemotherapy, Stearoyl-CoA desaturase 5 (SCD5)

Posted Date: December 10th, 2020

DOl: https://doi.org/10.21203/rs.3.rs-117096/v1

License: (c) (i) This work is licensed under a Creative Commons Attribution 4.0 International License. Read Full License 
Version of Record: A version of this preprint was published at Scientific Reports on April 26th, 2021. See the published version at https://doi.org/10.1038/s41598-021-88258-9. 


\section{Abstract}

Neoadjuvant chemotherapy (NACT) represents a standard option for breast cancer. Unfortunately, about $55 \%$ to $80 \%$ of breast cancer patients do not have a favorable response to chemotherapy. Highly specific tumor biomarker that can predict the pathological response to neoadjuvant chemotherapy is lacking. Stearoyl-CoA desaturase 5 (SCD5) is an integral membrane protein of the endoplasmic reticulum that participates in lipid metabolism. However, the role of SCD 5 in breast cancer remains unclear. Our study aims to understand its expression signature, prognosis value and correlation with pathological response to NACT in breast cancer using public databases. Analysis of samples from public databases showed that SCD 5 expression was down-regulated across human cancers and associated with more aggressive breast cancer phenotypes. Survival analysis revealed that SCD5 expression was related to prognosis in breast cancer, especially triple-negative breast cancer (TNBC). Integrated analysis of multiple public datasets indicated that SCD 5 expression signature was associated with response to NACT, particularly in TNBC. Based on functional enrichment analysis, SCD5 was implicated in pathways involved in metabolism and cell cycle. SCD5-related biological functions included negative regulation of cell cycle, cell division and DNA repair. Moreover, a significantly negative correlation between SCD5 expression and several cell cycle regulators was noted. Taken together, SCD5 was involved in the development and progression of breast cancer and might be a predictive biomarker for response to NACT. These results provided information for us to better understand SCD 5 from the perspective of bioinformatics and highlighted the clinical importance of SCD 5 in breast cancer, especially TNBC.

\section{Key Points}

1. SCD5 expression is correlated with survival in breast cancer.

2. SCD 5 could be a predictor of response to neoadjuvant chemotherapy in breast cancer, especially in TNBC

Subject terms: breast cancer, triple-negative breast cancer, pathological complete response, neoadjuvant chemotherapy, SCD5, signaling pathways

\section{Introduction}

Breast cancer is the most frequent cancer as well as the leading cause of cancer death among females. ${ }^{1}$ Breast cancer that lacks the expression of estrogen receptor (ER), progesterone receptor (PR), and human epidermal growth factor receptor 2 (HER2) is classified as TNBC. Compared to other breast cancers, TNBC accounts for $15 \%$ of all breast cancers and is associated with stronger invasiveness, higher risk of early recurrence rate and inferior survival. ${ }^{2}$ NACT, as a preoperative treatment to downgrade the tumors, was first used for locally advanced and inoperable breast cancer and now it has been widely used in operable breast cancer, especially in TNBC. Pathological complete response (pCR) is defined as the absence of residual invasive cancer on pathological evaluation of breast primary and lymphonodes after neoadjuvant chemotherapy. ${ }^{3}$ Several neodjuvant studies have revealed that pCR could predict long-term 
survival. ${ }^{4-6}$ The prognostic value of pathologic response makes NACT become the current standard option for patients with breast cancer. However, not all of patients with breast cancer have a favorable pathological response to NACT. ${ }^{7}$ It is well recognized that some intrinsic molecular and clinicopathological characteristics of tumor biology can affect the response degree to neoadjuvant chemotherapy. However, the highly specific biomarkers that can predict the pathological response to NACT and the prognosis of breast cancer have not been clearly defined.

Increased lipogenesis is one of most important metabolic markers of cancer cell. Cancer cell proliferation requires the continuous formation of new cell membranes, and the process of membrane lipid formation depends on the synthesis of fatty acids. ${ }^{8}$ The fatty acids composition of cell lipids is mainly regulated by stearoyl-CoA desaturase (SCDs). SCDs, as the endoplasmic reticulum-resident integral membrane proteins, catalyze the formation of monounsaturated fatty acids from saturated fatty acids. Four SCD isoforms (SCD1-SCD4) exist in mouse. However, only two SCD isoforms (SCD1 and SCD5) have been identified in human. ${ }^{9}$ SCD1 is confirmed to be up-regulated in the majority of cancers and participates in cell cycle and tumor cell migration. ${ }^{10,11}$ Different from SCD1, the biological role of SCD 5 in human cancers remains unclear. Previous studies found that SCD5 could protect against malignancy and reverse the epithelial-mesenchymal-like process in melanoma. ${ }^{12,13}$ In addition, Chen et al. found that the SCD5 expression in patients who achieved PCR after neoadjuvant chemotherapy was lower than that in patients who did not achieve pCR. ${ }^{14}$ Here, this study was performed to comprehensively analyze SCD5 expression characteristics, prognostic value and correlation with pathological response to NACT for better understanding the clinical significance of SCD5 in breast cancer.

\section{Materials And Methods}

\section{NCBI Gene Expression Omnibus (GEO)}

GEO (http://www.ncbi.nlm.nih.gov/geo/) is a worldwide resource for gene expression studies. It is used to archive and distribute high-throughput gene expression and other functional genomics data sets. ${ }^{15}$ GEO2R (https://www.ncbi.nlm.nih.gov/geo/geo2r/) and the software R (The R Programming Language, version 4.0.2) were performed to process the downloaded data. Then, the data was calibrated, standardized by software R.

\section{Oncomine}

The Oncomine platform (https://www.oncoline.org/; version 4.5), a cancer microarray database and webbased data-mining platform, aims at facilitating discovery from genome-wide expression analyses and compare the transcriptome data in most major types of cancer with normal counterparts. ${ }^{16}$ To date, Oncomine contains gene expression profile data of 86733 samples from 715 datasets.

Gene expression-based outcome for breast cancer online (GOBO) 
GOBO (http://co.bmc.lu.se/gobo『version 1.0.3) is an online analysis tool that offers the possibility of investigation of gene expression levels in breast cancer subgroups and breast cancer cell lines for gene sets. ${ }^{17}$

\section{UALCAN}

UALCAN (http://ualcan.path.uab.edu), as a portal for facilitating tumor subgroup gene expression and survival analyses, is an easy to use, interactive web-portal to perform to analysis of TCGA gene expression data. ${ }^{18}$

\section{Kaplan-Meier plotter}

The Kaplan-Meier-plotter online software (http://kmplot.com/analysis/) is an online database including gene expression data and clinical data. The prognostic value of the mRNA expression of SCD5 in breast cancer was evaluated using the Kaplan-Meier plotter. ${ }^{19}$

\section{Functional enrichment and gene ontology analysis}

The significant pathways influenced by SCD5 were analyzed by using KEGG pathway database (https://www.kegg.jp). Software R (version 4.0.2) was used to find SCD5-related DEGs. The functional enrichment and GO annotations of overlapped DEGs were analyzed by Metascape database (http://metascape.org).

\section{Statistical analysis}

The Wilcoxon rank-sum and t tests were used to analyze the association between gene expression and clinicopathological characteristics. Pearson correlation was performed to analysis the correlation between SCD5 and HER2/ERBB2 expression. Software R and GraphPad Prism (version 8.0.1) were applied for statistical analysis and image production. $P$ value $<0.05$ was determined to be significantly different (two-sided).

\section{Results}

\section{The mRNA expression of SCD5 in breast cancer}

To explore the roles of SCD5 in human cancers, we used the data samples from Oncomine, GEO and UALCAN to investigate altered SCD5 expression in normal tissue relative to cancer tissue. The analysis of multiple datasets showed that the SCD5 expression in human cancers was significantly down-regulated compared with their normal counterparts (Table S1, Figure 1A-B). In addition, we also analyzed the 
expression of SCD 5 in breast cancer patients with different molecular and clinicopathological characteristics. The results revealed that SCD5 expression was reduced in high histological grade and late stage breast cancer (Figure 1C-E). According to the expression status of ER, PR and HER2, the primary breast tumor was divided into different subtypes with different outcomes and response to treatment ${ }^{20}$. Two microarray datasets (GSE22226 and GSE20271) were downloaded from GEO to explore the distribution of SCD5 expression between TNBC and other breast cancer subtypes. Compared to other breast cancer subtypes, elevated SCD 5 expression was observed in TNBC (log2[Fold change] $>0.5, p<0.05$, Figure 2A-C). Due to the high intratumoral heterogeneity of breast cancer, only detecting the expression of ER, PR and HER2 is difficult to overcome the differences between patients and achieve precision medicine. Therefore, Parker et al. derived a minimal gene set (PAM50) to classify intrinsic subtypes of breast cancer in $2009^{21}$. According to differences in gene expression, breast cancer can be divided into five molecular subtypes (luminal A, luminal B, HER2 enriched, basal-like and normal-like). We further investigated the varied mRNA expression of SCD5 among five molecular subtypes in two GEO cohorts (GSE25065 and GSE22358). Basal-like breast cancer had the highest SCD5 expression compared to patients with other breast cancer subtypes, however, the SCD5 expression in HER2 enriched breast cancer was the lowest among five molecular subtypes ( $p<0.05$, Figure 2D-E). Taken together, these findings indicated that reduced SCD 5 expression was related to more aggressive breast cancer phenotypes, such as high histological grade, late stage and HER2 overexpressed breast cancer.

\section{Correlation analysis between mRNA expression of SCD5 and prognostic value}

We further explored the correlation between the gene expression and survival in breast cancer to better understand the clinical significance of SCD5. The Kaplan-Meier plotter, GOBO and Oncomine dataset (Loi3 breast dataset) were performed to carry out the survival analysis of breast cancer patients. As shown in Figure 3A, high expression of SCD 5 was related to decreased risk of relapse (HR=0.68 $95 \% \mathrm{Cl}=0.61$ to $0.76, \mathrm{p}=5.5 \mathrm{e}-12$ ). Among patients with TNBC, high SCD 5 expression cohort had a significantly $43 \%$ decreased risk of relapse compared with low SCD 5 expression cohort (Figure 3C, $\mathrm{HR}=0.5795 \% \mathrm{Cl}=0.44$ to $0.74, \mathrm{p}=1.3 \mathrm{e}-05)$. We also observed that SCD5 expression was higher in nonrecurrence group than that in recurrence group (Figure $3 \mathrm{E}, \mathrm{p}=0.01$ ). Besides, varied SCD 5 expression was correlated to DMFS in TNBC (Figure $3 \mathrm{C}, \mathrm{HR}=0.5295 \% \mathrm{Cl}=0.31$ to $0.89, \mathrm{p}=0.015$ ), which was not observed in either total breast tumors or other breast cancer subtypes (Figure $3 B$ and Figure $A 1, p>0.05$ ). The multivariate analysis showed that in patients with PAM50_basal-like breast tumors, lower SCD5 expression was related to higher risk of relapse (Figure 3F, p<0.05). SCD 5 expression was positively correlated to survival, especially in TNBC breast cancer patients.

\section{Relationship between mRNA expression of SCD5 and pathological response to neoadjuvant chemotherapy}


HER2/ERBB2 (erb-b2 receptor tyrosine kinase 2), a member of the epidermal growth factor receptor family, is amplified/overexpressed in 15 to $20 \%$ of breast cancers and associated with a poor prognosis. ${ }^{22}$ Some correlative studies of breast cancer indicated that the overexpression/amplification of HER2/ERBB2 was associated with a significant benefit from paclitaxel/doxorubicin. ${ }^{23-26}$ Thus, patients with HER2-negative might benefit relatively less from NACT. Besides, there is still no clinically useful test for prediction of response or survival following chemotherapy for breast cancer. Thus, finding new biomarkers to predict the response of NACT is imperative. Previous study showed that the expression of SCD 5 varied between pCR cohort and non-pCR cohort ${ }^{14}$. In this article, we have already found that the SCD 5 expression in triple-negative breast cancer was higher than that in other breast cancer subtypes using public datasets. Then, we further explored the relationship between SCD 5 expression and HER2/ERBB2 expression in Oncomine (Gluck breast cancer) and GEO (GSE20194) datasets. As shown in Figure A3, SCD5 expression was negatively correlated HER2/ERBB2 expression $(p<0.05)$. Thus, we speculated that high SCD5 expression characteristic was associated with unfavorable response to chemotherapy in breast cancer. Therefore, we performed microarray datasets downloaded from GEO (GSE20194, GSE20271 and GSE25055) and Oncomine (Garnett cell line) to further define the value of SCD 5 in curative effect assessment of anthracycline/taxane-based NACT for breast cancer. Analysis results showed that SCD5 was differentially expressed between responders and non-responders. The SCD 5 exhibited a significant low expression in responders in comparison to non-responders (Figure 4A-B $\mathrm{p}<0.05)$. We also used TNBC samples from GSE20271 datasets to analyze the pathological responserelated differentially expressed genes. The volcano plot and heat map showed that SCD5 expression was significantly up-regulated in non-pCR group compared with pCR group (Figure A2). Besides, we found that the fold change of differential SCD5 expression in TNBC was higher than that in total tumors.

Furthermore, receiver operating characteristic curve (ROC curve) analysis indicated that SCD 5 could be a good predictor of pCR in patients with breast cancer, especially in TNBC (Figure 4C-D, Table 1, p<0.05). In GEO dataset (GSE25055), patients were divided to chemosensitive and chemo-resistant groups according to different pathological responses to taxane-anthracycline based NACT. The SCD5 expression was significantly reduced in chemosensitive group compared with chemo-resistant group $(p=0.001$, log2 [Fold change] $=-0.57$ ). Figure $4 \mathrm{~F}$ showed that in different cancer cell lines, SCD 5 exhibited a high expression in paclitaxel resistant group compared to paclitaxel sensitive group $(p=0.05)$. 
Table 1

ROC analysis of SCD5 in pretreatment tumor biopsy samples for predicting pCR

\begin{tabular}{|c|c|c|c|c|c|c|}
\hline \multirow[t]{2}{*}{ GEO datasets } & \multicolumn{3}{|c|}{ No. of patients } & \multirow[t]{2}{*}{ AUC } & \multirow[t]{2}{*}{$P$ value } & \multirow[t]{2}{*}{$95 \% \mathrm{Cl}$} \\
\hline & Total & $\begin{array}{l}\text { No. of } \\
\text { pCR }\end{array}$ & $\begin{array}{l}\text { No. of non- } \\
\text { pCR }\end{array}$ & & & \\
\hline $\begin{array}{l}\text { GSE20194(Total } \\
\text { tumors) }\end{array}$ & 278 & 56 & 222 & 0.6123 & 0.0094 & $\begin{array}{l}0.5317 \text { to } \\
0.6929\end{array}$ \\
\hline $\begin{array}{l}\text { GSE20194(TNBC } \\
\text { tumors) }\end{array}$ & 71 & 25 & 46 & 0.6817 & 0.0119 & $\begin{array}{l}0.5496 \text { to } \\
0.8139\end{array}$ \\
\hline $\begin{array}{l}\text { GSE20271(Total } \\
\text { tumors) }\end{array}$ & 178 & 54 & 124 & 0.6441 & 0.0023 & $\begin{array}{l}0.5612 \text { to } \\
0.7271\end{array}$ \\
\hline $\begin{array}{l}\text { GSE20271(TNBC } \\
\text { tumors) }\end{array}$ & 59 & 27 & 32 & 0.8368 & $<0.0001$ & $\begin{array}{l}0.7329 \text { to } \\
0.9407\end{array}$ \\
\hline
\end{tabular}

\section{Potential pathways influenced by SCD5}

In the previous section, we mentioned that the SCD5 expression was down-regulated in breast cancer compared to normal breast tissue and was negatively correlated to ERBB2/HER2 expression $(\mathrm{p}<0.05)$. Besides, we also found that the compared with wild type, SCD 5 expression was down-regulated in several oncogene/tumor suppression genes mutation type (Figure A4) such as KRAS (Kirsten Rat Sarcoma Viral Oncogene Homolog), BRAF (B-Raf proto-oncogene, serine/threonine kinase and CDKN2A (cyclin dependent kinase inhibitor $2 A$ ). Therefore, we speculated that SCD5 might play a role in inhibiting the development and progression of tumor cells. KEGG pathway database and Metascape database were used to further explore the potential SCD 5 regulatory mechanisms. Analysis of KEGG pathway showed that besides fatty acid metabolic pathway, SCD 5 was also involved in peroxisome proliferator-activated receptor (PPAR) and AMP-activated protein kinase (AMPK) signaling pathways (Table 2), which were related to lipid metabolism as well as the inhibition of the multiplication of tumor cell. ${ }^{27-30}$ In addition, AMPK was also closely involved in cancer drug resistance. ${ }^{31}$

Table 2

KEGG pathway of SCD5

\begin{tabular}{|lll|}
\hline Pathway & Description & p value \\
\hline hsa01040 & Biosynthesis of unsaturated fatty acids & 0.003 \\
\hline hsa01212 & Fatty acid metabolism & 0.007 \\
\hline hsa03320 & PPAR signaling pathway & 0.009 \\
\hline hsa04152 & AMPK signaling pathway & 0.01 \\
\hline
\end{tabular}


To further study the possible molecular functions and biological networks of SCD5, two GEO cohorts samples (GSE25055 and GSE25065) were divided into two groups according to high SCD5 expression (top 20\%) and low SCD5 expression (bottom 20\%). A total of 1923 genes (1315 down-regulated and 608 up-regulated) that had a $\mid \log 2$ (fold change) $\mid \geq 1$ and a p-value $<0.05$ were considered significantly differentially expressed. Biological annotations and functional enrichment of overlapped DEGs were performed by using Metascape database. The results of GO analysis (Figure 5) showed that the 608 SCD5-related DEGs (up-regulated in breast cancer with high SCD 5 expression) were involved in negative regulation of cell cycle (the most significant biological process). In addition, these DEGs were also involved in cell division, DNA repair and so on. SCD5, by and large, might act as a tumor suppression gene and negatively regulate cancer cell growth and proliferation in breast cancer.

In order to further understand the role of SCD5 in cell cycle, we explored correlations between SCD5 expression and several cell cycle regulators using dataset from Oncomine (Hatzis Breast). Most of the known cyclin-dependent kinases (CDKs) could regulate cell cycle progression. There are at least nine isoforms of CDKs in animal cells, among which four isoforms (CDK1-4) are directly involved in the regulation of cell cycle. In mammalian cells, CDK1 interacts with cyclin to drive cell cycle progression. Another isoform, CDK7, is indirectly involved in the activation of cyclin dependent kinase activated kinase. ${ }^{32}$ Analyses revealed that SCD 5 expression was negatively correlated with multiple cell cycle regulators (Table S2).

\section{Discussion}

Currently, NACT has become a major trend in clinical management of breast cancer. Improved response to NACT is related to better survival outcomes. However, there is still a lack of effective methods to select breast cancer patients who would benefit from NACT. Therefore, it is necessary to identify novel biomarkers that may predict response to NACT. Previous study showed that the SCD 5 expression in patients who achieved PCR after neoadjuvant chemotherapy was lower than that in patients who did not achieve pCR. ${ }^{14}$ Here, we used public datasets and revealed that SCD5 could be a potential predictor of response to neoadjuvant therapy, especially for TNBC.

Dysregulation of lipid metabolism is considered as a component of malignant transformation in many different cancers, including breast cancer. ${ }^{33}$ The balance between saturated and monounsaturated fatty acids could regulate of cell growth and differentiation through affecting cell membrane fluidity and signal transduction. ${ }^{34}$ SCDs was known as the rate-limiting enzyme catalyzing the synthesis of monounsaturated fatty acids. ${ }^{35,36}$ Two isoforms of SCDs (SCD1 and SCD5) are characterized in human. ${ }^{9}$ It is well recognized that SCD1 expression is up-regulated in cancer cells which could promote the cell proliferation and increase tumor membrane fluidity. ${ }^{10}$ Different from the up-regulated expression of SCD1 in human cancers, our data revealed that SCD5 was reduced in multiple cancer tissues compared to their normal counterparts. Furthermore, we found that reduced SCD5 expression was correlated with some aggressive features, such as high histological grade, late stage, the HER2 overexpressed subtype, BRAF, 
KRAS and CDKN2A mutations, higher recurrence rate, as well as shorter DMFS and RFS. Analysis of KEGG pathway showed that SCD5 was involved in peroxisome proliferator-activated receptor (PPAR) and AMP-activated protein kinase (AMPK) signaling pathways (Table 2), which were related to the inhibition of the multiplication of tumor cell. Results of $\mathrm{GO}$ annotation and functional enrichments showed that SCD5-related DEGs were mainly involved in negative regulation of cell cycle cell division and DNA repair. These findings are similar to observations in melanoma. Bellenghi $\mathrm{M}$ et al proved that the restored expression of SCD 5 and its production- oleic acid could protect against malignancy in melanoma, especially during the metastatic dissemination phase by driving advanced melanoma cell lines toward differentiation and reversion of epithelial-mesenchymal. ${ }^{12,13}$ We further analyzed the correlation between HER2 and SCD5 expression and observed SCD 5 expression was negatively correlated to HER2 expression. The former research confirmed that monounsaturated fatty acid could suppress HER2 expression in breast cancer, ${ }^{37}$ which might explain the negative correlation between SCD 5 and HER2 expression as well as the higher SCD 5 expression in TNBC compared with other molecular subtypes. Stated thus, these data suggested that SCD5 might function as a regulator of diverse signaling networks to suppress cell proliferation and migration in breast cancer.

Moreover, our data also suggested that SCD5 could be a potential predictor of response to anthracycline/taxane-based neoadjuvant chemotherapy in patients with breast cancer, especially in TNBC patients. Our data showed that SCD5 expression was negatively correlated with multiple cell cycle regulators. The clinical used cytotoxic drugs such as paclitaxel and vinblastine are cell cycle specific agents (CCSA), which are only sensitive to certain phases of the cell proliferation cycle, but not to G0 phase cells. Thus, we hypothesized that high SCD5 expression might induce cell cycle checkpoint arrest and lead to chemoresistance. In addition, Wang et al. observed that fatty acid oxidation rate was significantly increased in paclitaxel-resistant TNBC (MDA-MB 231) cells and indicated that fatty acids oxidation was required for the maintenance of breast cancer stem cells and contributes to chemoresistance. ${ }^{38}$ Because of the absence of double bonds, saturated fatty acids are the most stable among fatty acids. With the increase of unsaturated double bond, the fatty acids become more unstable and easier to be oxidized. ${ }^{39}$ We speculated that SCD5 might catalyze the formation of unsaturated fatty acids from saturated fatty acids, which increase the fatty acid oxidation rate and thus lead to chemoresistance. The mechanism of this part needs further study. In addition, our data observed that SCD5 was significantly involved in AMPK signaling pathway, which was related to cancer drug resistance. ${ }^{31}$ Taken together, SCD5 expression signatures could be associated with response of neoadjuvant chemotherapy in breast cancer.

In summary, our study was the first to investigate the roles of ILF2 in breast cancer, particularly in TNBC. The results showed that SCD5 might function as a tumor suppressor gene during breast cancer progression. Furthermore, SCD5 might help distinguish breast cancer patients, especially TNBC patients, who are likely to benefit from neoadjuvant chemotherapy.

\section{Declarations}




\section{Acknowledgements}

This work was supported by the following grants: Key Task Project of Tianjin Health and Family Planning Commission (16KG128), Anticancer Key Technologies R\&D Program of Tianjin (12ZCDZSY16200) and Natural Science Foundation of Tianjin (18JCYBJC91600).

\section{Author Contributions}

P.Z. conceived the research design. P.Z., L.S. and Z.T. were the main contributors of manuscript text, figures and table. P.Z., L.S., X.L., J.W., Y.Z. and Y.J. contributed the analysis of data. All authors reviewed the manuscript, provided edits and suggestions and approved the final manuscript.

\section{Data Availability}

All data is provided in the manuscript.

\section{Competing interests}

The author(s) declare no competing interests.

\section{Ethical standards}

All data in this manuscript was collected under the guidelines approved by Tianjin Medical University Cancer Institute and Hospital's institutional review board and complying with the current laws in China.

\section{References}

1. Erratum: Global cancer statistics 2018: GLOBOCAN estimates of incidence and mortality worldwide for 36 cancers in 185 countries. CA Cancer J Clin70, 313, doi:10.3322/caac.21609 (2020).

2. Brown, M., Tsodikov, A., Bauer, K. R., Parise, C. A. \& Caggiano, V. The role of human epidermal growth factor receptor 2 in the survival of women with estrogen and progesterone receptor-negative, invasive breast cancer: the California Cancer Registry, 1999-2004. Cancer112, 737-747, doi:10.1002/cncr.23243 (2008).

3. von Minckwitz, G. et al. Definition and impact of pathologic complete response on prognosis after neoadjuvant chemotherapy in various intrinsic breast cancer subtypes. J Clin Onco/30, 1796-1804, doi:10.1200/JC0.2011.38.8595 (2012).

4. Liedtke, C. et al. Response to neoadjuvant therapy and long-term survival in patients with triplenegative breast cancer. J Clin Onco/26, 1275-1281, doi:10.1200/JC0.2007.14.4147 (2008). 
5. Cortazar, P. et al. Pathological complete response and long-term clinical benefit in breast cancer: the CTNeoBC pooled analysis. Lancet384, 164-172, doi:10.1016/S0140-6736(13)62422-8 (2014).

6. Kong, X., Moran, M. S., Zhang, N., Haffty, B. \& Yang, Q. Meta-analysis confirms achieving pathological complete response after neoadjuvant chemotherapy predicts favourable prognosis for breast cancer patients. Eur J Cancer47, 2084-2090, doi:10.1016/j.ejca.2011.06.014 (2011).

7. Early Breast Cancer Trialists' Collaborative, G. Long-term outcomes for neoadjuvant versus adjuvant chemotherapy in early breast cancer: meta-analysis of individual patient data from ten randomised trials. Lancet Onco/19, 27-39, doi:10.1016/S1470-2045(17)30777-5 (2018).

8. Zaidi, N. et al. Lipogenesis and lipolysis: the pathways exploited by the cancer cells to acquire fatty acids. Prog Lipid Res52, 585-589, doi:10.1016/j.plipres.2013.08.005 (2013).

9. Wang, J. et al. Characterization of HSCD5, a novel human stearoyl-CoA desaturase unique to primates. Biochem Biophys Res Commun332, 735-742, doi:10.1016/j.bbrc.2005.05.013 (2005).

10. Scaglia, N. \& Igal, R. A. Stearoyl-CoA desaturase is involved in the control of proliferation, anchorageindependent growth, and survival in human transformed cells. J Biol Chem280, 25339-25349, doi:10.1074/jbc.M501159200 (2005).

11. Angelucci, C. et al. Pivotal role of human stearoyl-CoA desaturases (SCD1 and 5) in breast cancer progression: oleic acid-based effect of SCD1 on cell migration and a novel pro-cell survival role for SCD5. Oncotarget9, 24364-24380, doi:10.18632/oncotarget.25273 (2018).

12. Bellenghi, M. et al. SCD5-induced oleic acid production reduces melanoma malignancy by intracellular retention of SPARC and cathepsin B. J Patho/236, 315-325, doi:10.1002/path.4535 (2015).

13. Puglisi, R. et al. SCD5 restored expression favors differentiation and epithelial-mesenchymal reversion in advanced melanoma. Oncotarget9, 7567-7581, doi:10.18632/oncotarget.24085 (2018).

14. Chen, Y. Z. et al. PPAR signaling pathway may be an important predictor of breast cancer response to neoadjuvant chemotherapy. Cancer Chemother Pharmaco/70, 637-644, doi:10.1007/s00280-0121949-0 (2012).

15. Clough, E. \& Barrett, T. The Gene Expression Omnibus Database. Methods Mol Bio/1418, 93-110, doi:10.1007/978-1-4939-3578-9_5 (2016).

16. Rhodes, D. R. et al. ONCOMINE: a cancer microarray database and integrated data-mining platform. Neoplasia6, 1-6, doi:10.1016/s1476-5586(04)80047-2 (2004).

17. Ringner, M., Fredlund, E., Hakkinen, J., Borg, A. \& Staaf, J. GOBO: gene expression-based outcome for breast cancer online. PLoS One6, e17911, doi:10.1371/journal.pone.0017911 (2011).

18. Chandrashekar, D. S. et al. UALCAN: A Portal for Facilitating Tumor Subgroup Gene Expression and Survival Analyses. Neoplasia19, 649-658, doi:10.1016/j.neo.2017.05.002 (2017).

19. Hou, G. X., Liu, P., Yang, J. \& Wen, S. Mining expression and prognosis of topoisomerase isoforms in non-small-cell lung cancer by using Oncomine and Kaplan-Meier plotter. PLoS One12, e0174515, doi:10.1371/journal.pone.0174515 (2017). 
20. Perou, C. M. et al. Molecular portraits of human breast tumours. Nature406, 747-752, doi:10.1038/35021093 (2000).

21. Parker, J. S. et al. Supervised risk predictor of breast cancer based on intrinsic subtypes. J Clin Onco/27, 1160-1167, doi:10.1200/JC0.2008.18.1370 (2009).

22. Slamon, D. J. et al. Human breast cancer: correlation of relapse and survival with amplification of the HER-2/neu oncogene. Science235, 177-182, doi:10.1126/science.3798106 (1987).

23. Hayes, D. F. et al. HER2 and response to paclitaxel in node-positive breast cancer. N Engl J Med357, 1496-1506, doi:10.1056/NEJMoa071167 (2007).

24. Ueno, N. T., Yu, D. \& Hung, M. C. Chemosensitization of HER-2/neu-overexpressing human breast cancer cells to paclitaxel (Taxol) by adenovirus type 5 E1A. Oncogene15, 953-960, doi:10.1038/sj.onc.1201250 (1997).

25. Muss, H. B. et al. c-erbB-2 expression and response to adjuvant therapy in women with node-positive early breast cancer. N Engl J Med330, 1260-1266, doi:10.1056/NEJM199405053301802 (1994).

26. Dressler, L. G. et al. Comparison of HER2 status by fluorescence in situ hybridization and immunohistochemistry to predict benefit from dose escalation of adjuvant doxorubicin-based therapy in node-positive breast cancer patients. J Clin Onco/23, 4287-4297, doi:10.1200/JCO.2005.11.012 (2005).

27. Nickkho-Amiry, M., McVey, R. \& Holland, C. Peroxisome proliferator-activated receptors modulate proliferation and angiogenesis in human endometrial carcinoma. Mol Cancer Res10, 441-453, doi:10.1158/1541-7786.MCR-11-0233 (2012).

28. Li, W., Saud, S. M., Young, M. R., Chen, G. \& Hua, B. Targeting AMPK for cancer prevention and treatment. Oncotarget6, 7365-7378, doi:10.18632/oncotarget.3629 (2015).

29. Bougarne, N. et al. Molecular Actions of PPARalpha in Lipid Metabolism and Inflammation. Endocr Rev39, 760-802, doi:10.1210/er.2018-00064 (2018).

30. He, L. et al. AMPK Regulation of Glucose, Lipid and Protein Metabolism: Mechanisms and Nutritional Significance. Curr Protein Pept Sci18, 562-570, doi:10.2174/1389203717666160627071125 (2017).

31. Wang, Z., Wang, N., Liu, P. \& Xie, X. AMPK and Cancer. Exp Supp/107, 203-226, doi:10.1007/978-3319-43589-3_9 (2016).

32. Satyanarayana, A. \& Kaldis, P. Mammalian cell-cycle regulation: several Cdks, numerous cyclins and diverse compensatory mechanisms. Oncogene28, 2925-2939, doi:10.1038/onc.2009.170 (2009).

33. Baumann, J., Sevinsky, C. \& Conklin, D. S. Lipid biology of breast cancer. Biochim Biophys Acta1831, 1509-1517, doi:10.1016/j.bbalip.2013.03.011 (2013).

34. Ntambi, J. M. \& Miyazaki, M. Regulation of stearoyl-CoA desaturases and role in metabolism. Prog Lipid Res43, 91-104, doi:10.1016/s0163-7827(03)00039-0 (2004).

35. St John, L. C., Lunt, D. K. \& Smith, S. B. Fatty acid elongation and desaturation enzyme activities of bovine liver and subcutaneous adipose tissue microsomes. J Anim Sci69, 1064-1073, 
doi:10.2527/1991.6931064x (1991).

36. Rincon, G. et al. Polymorphisms in genes in the SREBP1 signalling pathway and SCD are associated with milk fatty acid composition in Holstein cattle. J Dairy Res79, 66-75, doi:10.1017/S002202991100080X (2012).

37. Menendez, J. A., Vellon, L., Colomer, R. \& Lupu, R. Oleic acid, the main monounsaturated fatty acid of olive oil, suppresses Her-2/neu (erbB-2) expression and synergistically enhances the growth inhibitory effects of trastuzumab (Herceptin) in breast cancer cells with Her-2/neu oncogene amplification. Ann Onco/16, 359-371, doi:10.1093/annonc/mdi090 (2005).

38. Wang, T. et al. JAK/STAT3-Regulated Fatty Acid beta-Oxidation Is Critical for Breast Cancer Stem Cell Self-Renewal and Chemoresistance. Cell Metab27, 136-150 e135, doi:10.1016/j.cmet.2017.11.001 (2018).

39. Leyton, J., Drury, P. J. \& Crawford, M. A. Differential oxidation of saturated and unsaturated fatty acids in vivo in the rat. Br J Nutr57, 383-393, doi:10.1079/bjn19870046 (1987).

\section{Figures}


Median Rank p-Value Gene

$2787.5 \quad 0.020 \quad$ SCD5

Legend

1. Invasive Ductal Breast Carcinoma vs.

Curtis Breast, Nature, 2012

2. Invasive Lobular Breast Carcinoma vs.

Curtis Breast, Nature, 2012

3. Mucinous Breast Carcinoma vs. Normal

Curtis Breast, Nature, 2012

4. Tubular Breast Carcinoma vs. Normal

Curtis Breast, Nature, 2012

5. Invasive Breast Carcinoma Stroma vs.

Finak Breast, Nat Med, 2008

6. Invasive Ductal Breast Carcinoma Stroma

vs. Normal

. Ductal Breast Carcinoma in Situ Epithelia

vs. Normal

ast Cancer Res, 2009

8. Ductal Breast Carcinoma in Situ vs. Norm

200

\section{\begin{tabular}{|l|l|l|l|l|l|l|l|l|l|l|l|l|l|l|}
\hline 1 & 2 & 3 & 4 & 5 & 6 & 7 & 8 & 9 & 10 & 11 & 12 & 13 & 14 & 15 \\
\hline
\end{tabular}}

9. Invasive Lobular Breast Carcinoma vs.

Normal
Radvanyi Breast, Proc Natl Acad Sci U S A, 2005

10. Invasive Ductal and Lobular Carcinoma vs. Normal

TCGA Breast, No Associated Paper, 2011

11. Invasive Ductal Breast Carcinoma vs.

NCGAA Breast, No Associated Paper, 2011

12. Invasive Lobular Breast Carcinoma vs.

TCGA Breast, No Associated Paper, 2011

13. Mixed Lobular and Ductal Breast Carcinoma vs. Normal

14. Invasive Lobular Breast Carcinoma vs.

Turashvili Breast, BMC Cancer, 2007

15. Invasive Ductal Breast Carcinoma vs. Normal

Zhao Breast, Mol Biol Cell, 2004

16. Lobular Breast Carcinoma vs. Normal

Zhoo Breast, Mol Biol Cell, 2004
B

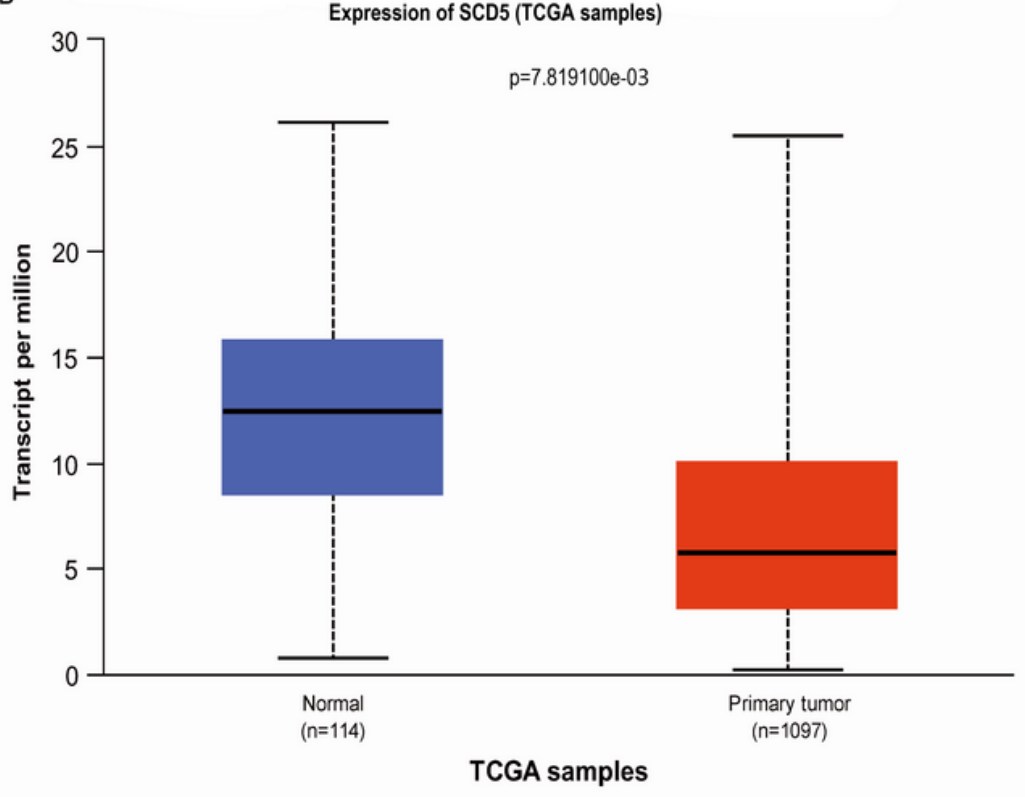

C

ㅁㅁㅁㅁㅁㅁㅁㅁํ

The rank for a gene is the median rank for that gene across each of the analyses.
The p-Value for a gene is its p-Value for the median-ranked analysis.

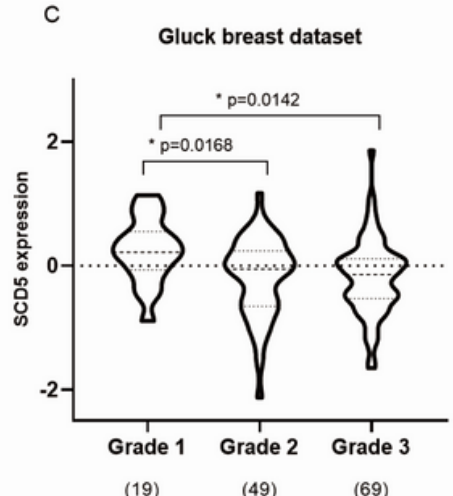

(19)
(49)

(69)
D

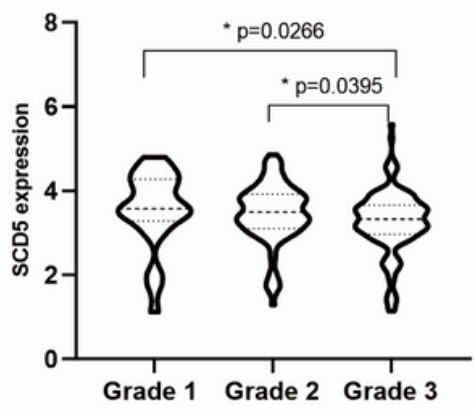

(30)

(107)

(141)

E

Boersma breast dataset

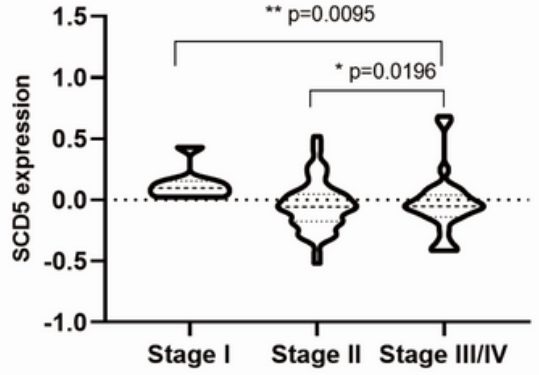

(8)

(28)

\section{Figure 1}

The mRNA expression of SCD5 in breast cancer. (A). SCD5 expression in breast tumor tissues compared with normal breast tissues. Meta-analysis of SCD5 expression in breast cancer by using dataset from Oncomine. $(P<0.05$ and fold change $>1.5)$. The colored squares indicate the median rank for SCD 5 across each analysis comparing breast cancer with normal counterparts. (B). SCD 5 expression in breast cancer compared with normal counterparts by using dataset from UALCAN. (C). SCD5 expression in different histological grades of breast cancer using Gluck breast dataset from Oncomine. (D). SCD5 expression in different histological grades of breast cancer using Bittner breast dataset from Oncomine. (E). SCD 5 expression in different stages of breast cancer using Boersma breast dataset from Oncomine. 
A

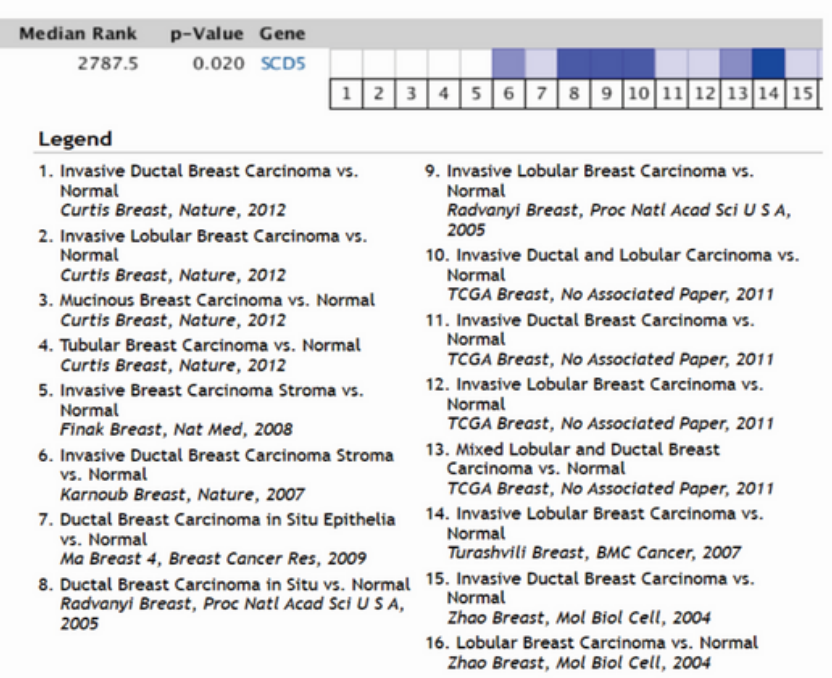

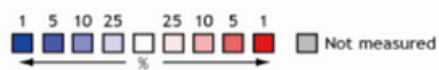

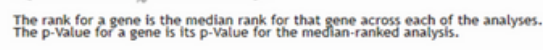

B

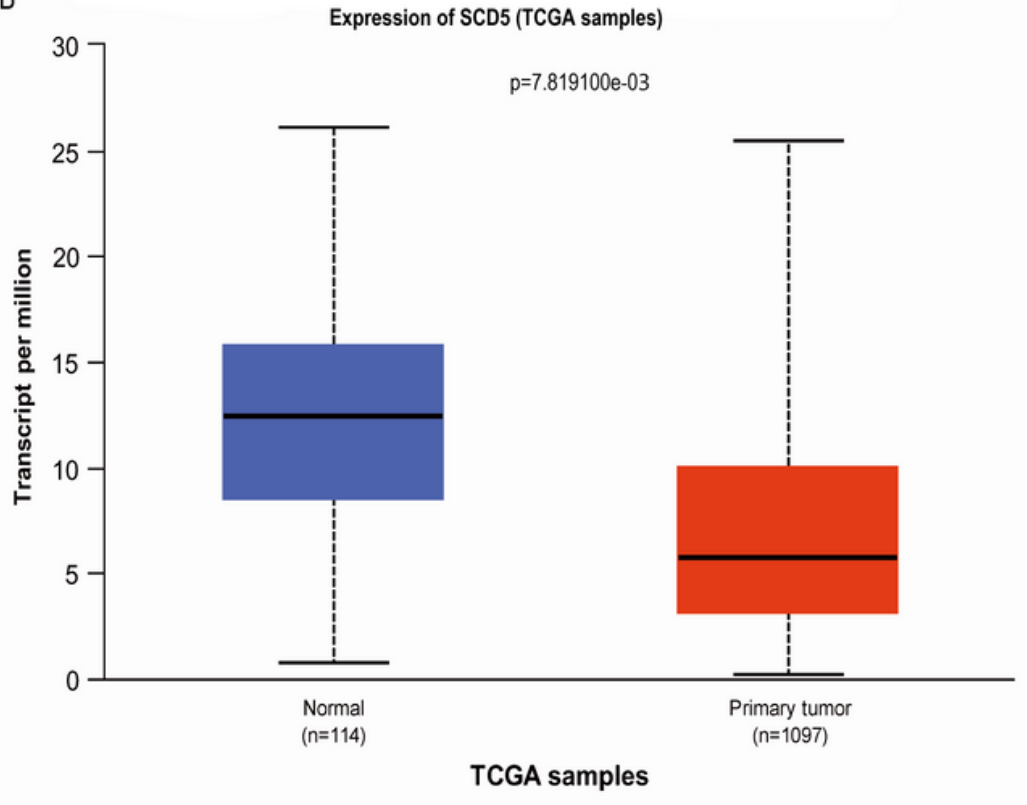

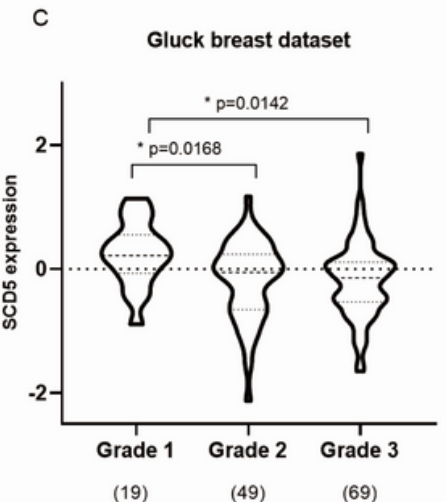

(19)

(49)

(69)
D

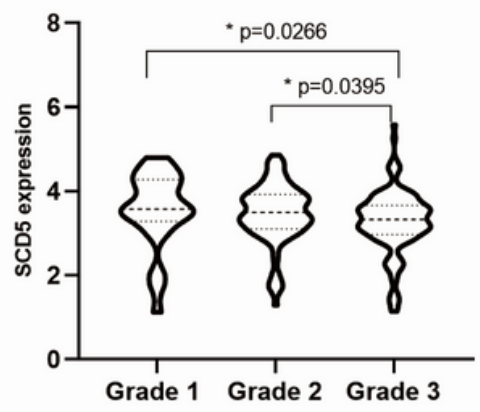

(30) (107) (141)
E

Boersma breast dataset

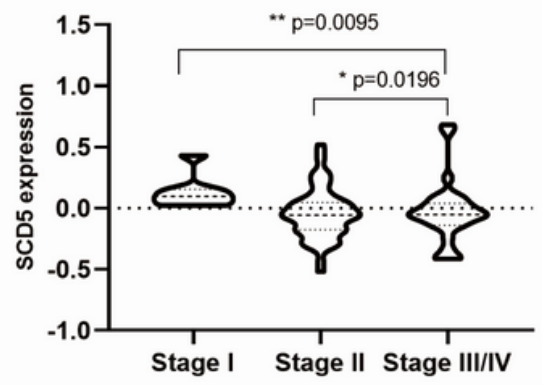

(8)

(59)

(28)

\section{Figure 1}

The mRNA expression of SCD 5 in breast cancer. (A). SCD5 expression in breast tumor tissues compared with normal breast tissues. Meta-analysis of SCD5 expression in breast cancer by using dataset from Oncomine. $(P<0.05$ and fold change $>1.5)$. The colored squares indicate the median rank for SCD 5 across each analysis comparing breast cancer with normal counterparts. (B). SCD5 expression in breast cancer compared with normal counterparts by using dataset from UALCAN. (C). SCD5 expression in different histological grades of breast cancer using Gluck breast dataset from Oncomine. (D). SCD5 expression in different histological grades of breast cancer using Bittner breast dataset from Oncomine. (E). SCD5 expression in different stages of breast cancer using Boersma breast dataset from Oncomine. 


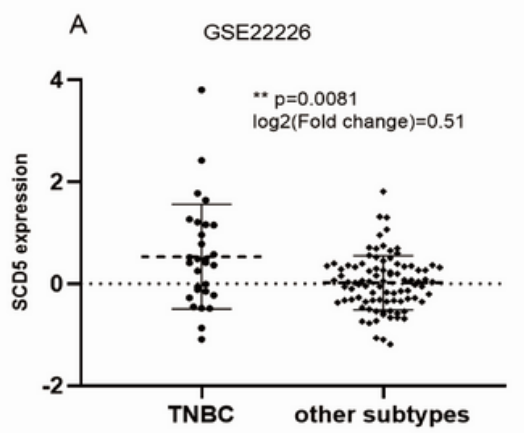

(29) (90)

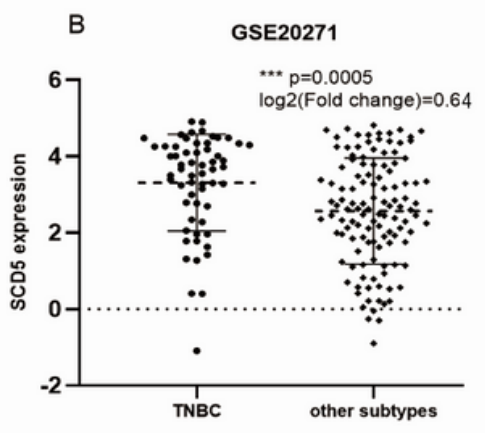

(59)

(119)
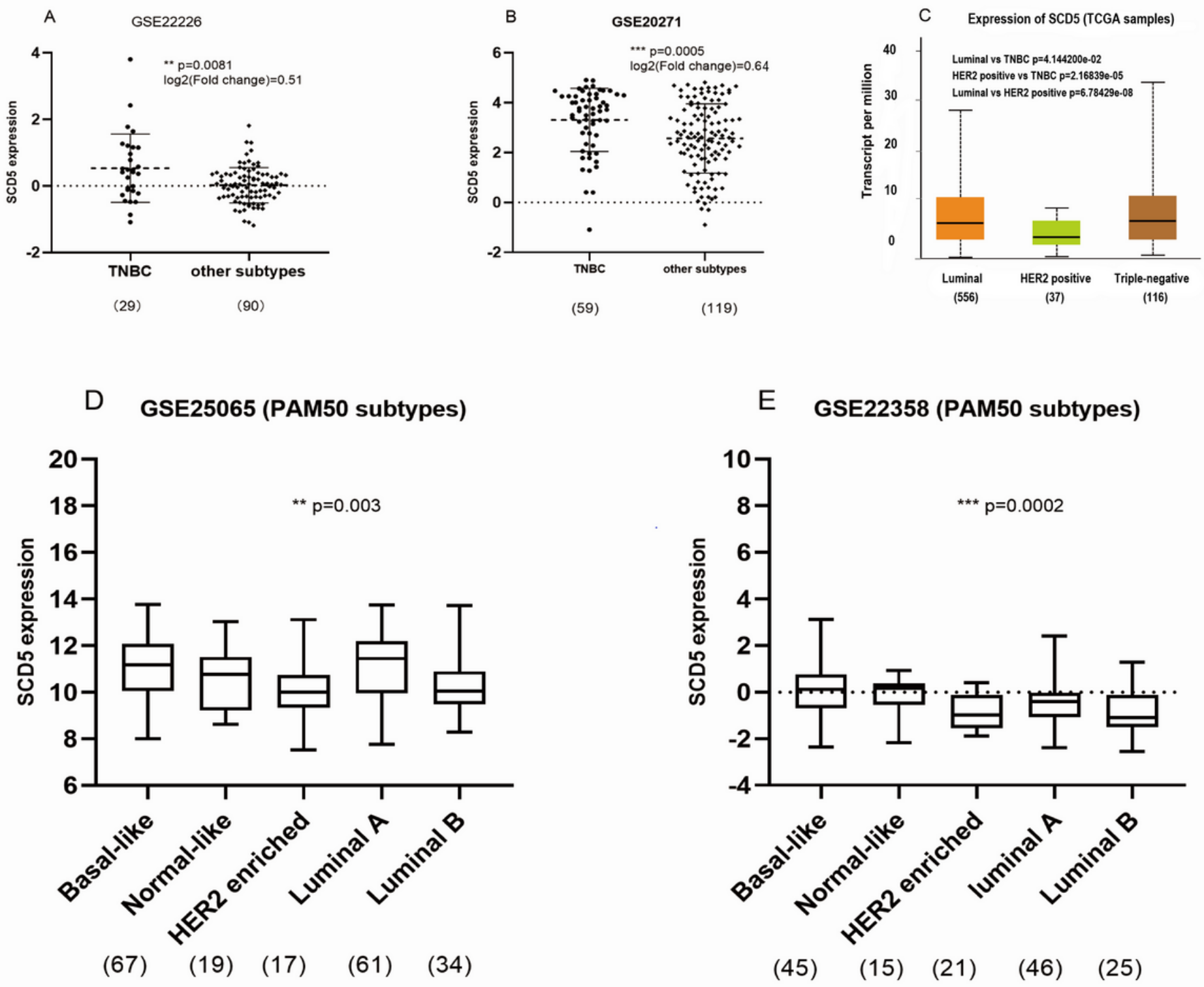

Figure 2

The mRNA expression of SCD5 in different molecular subtypes of breast cancer. (A-B). SCD5 expression in TNBC and other breast cancer using datasets from GEO. (C). SCD5 expression in luminal, HER2 positive and TNBC using dataset from UALCAN. (D-E). SCD5 expression in Basal-like, Normal-like, HER2 enriched, Luminal $A$ and Luminal $B$ using datasets from GEO. 


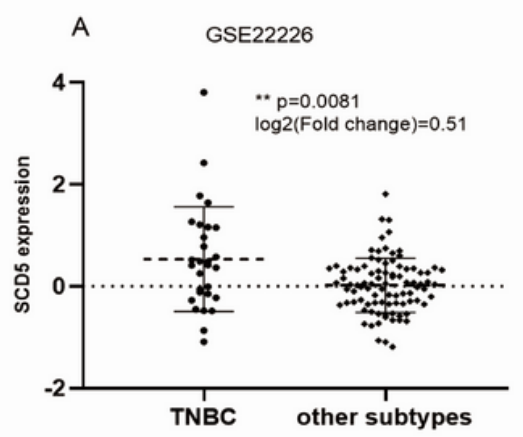

(29)

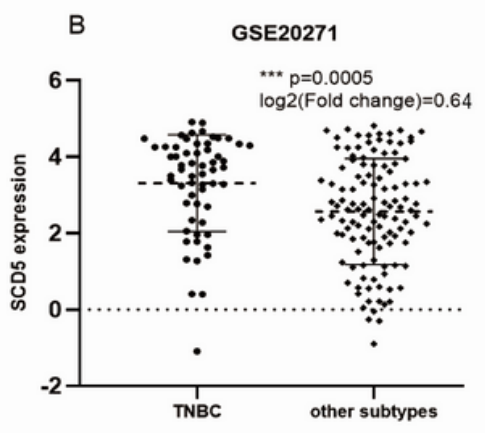

(59)

(119)
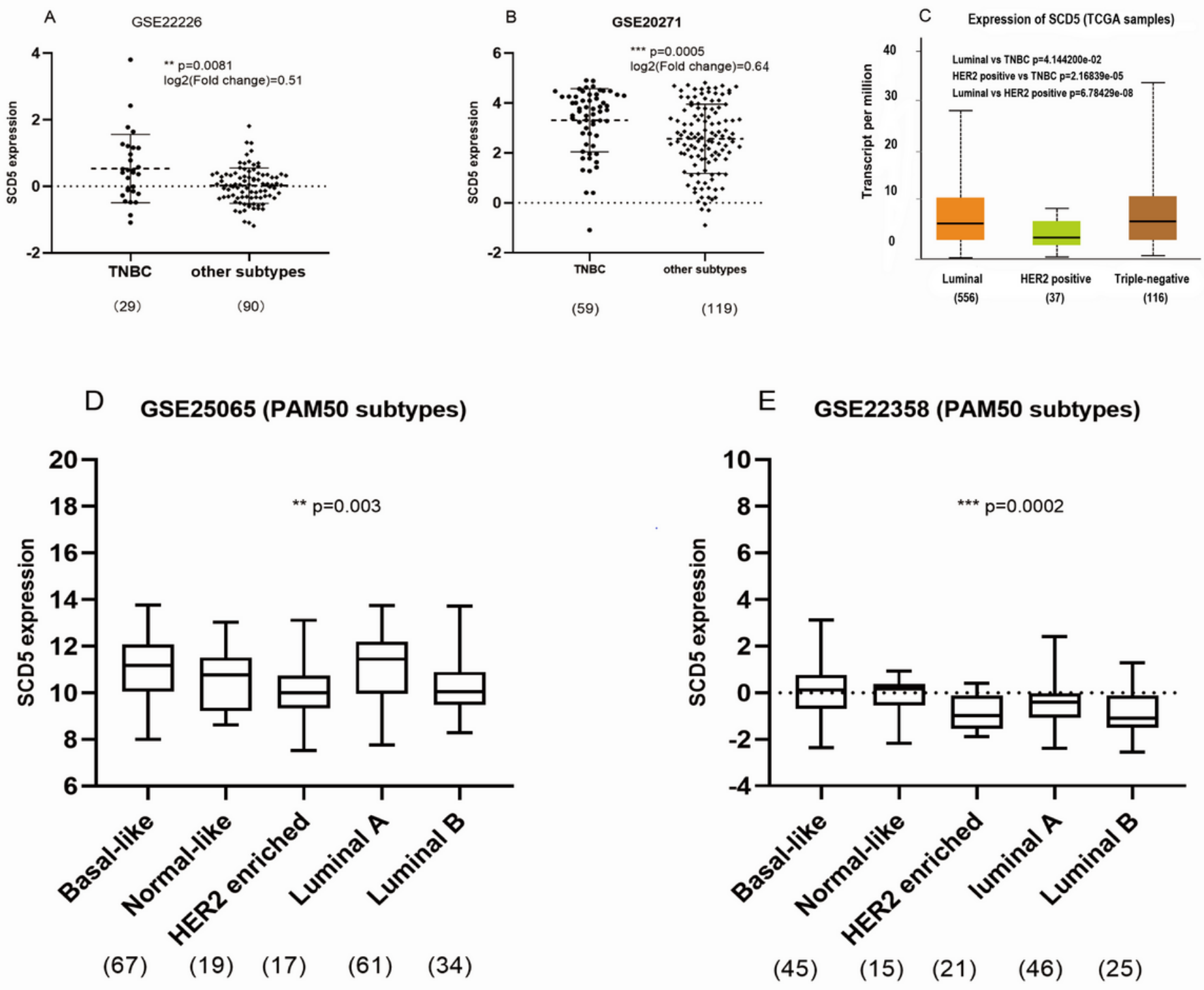

Figure 2

The mRNA expression of SCD5 in different molecular subtypes of breast cancer. (A-B). SCD5 expression in TNBC and other breast cancer using datasets from GEO. (C). SCD5 expression in luminal, HER2 positive and TNBC using dataset from UALCAN. (D-E). SCD5 expression in Basal-like, Normal-like, HER2 enriched, Luminal $A$ and Luminal $B$ using datasets from GEO. 

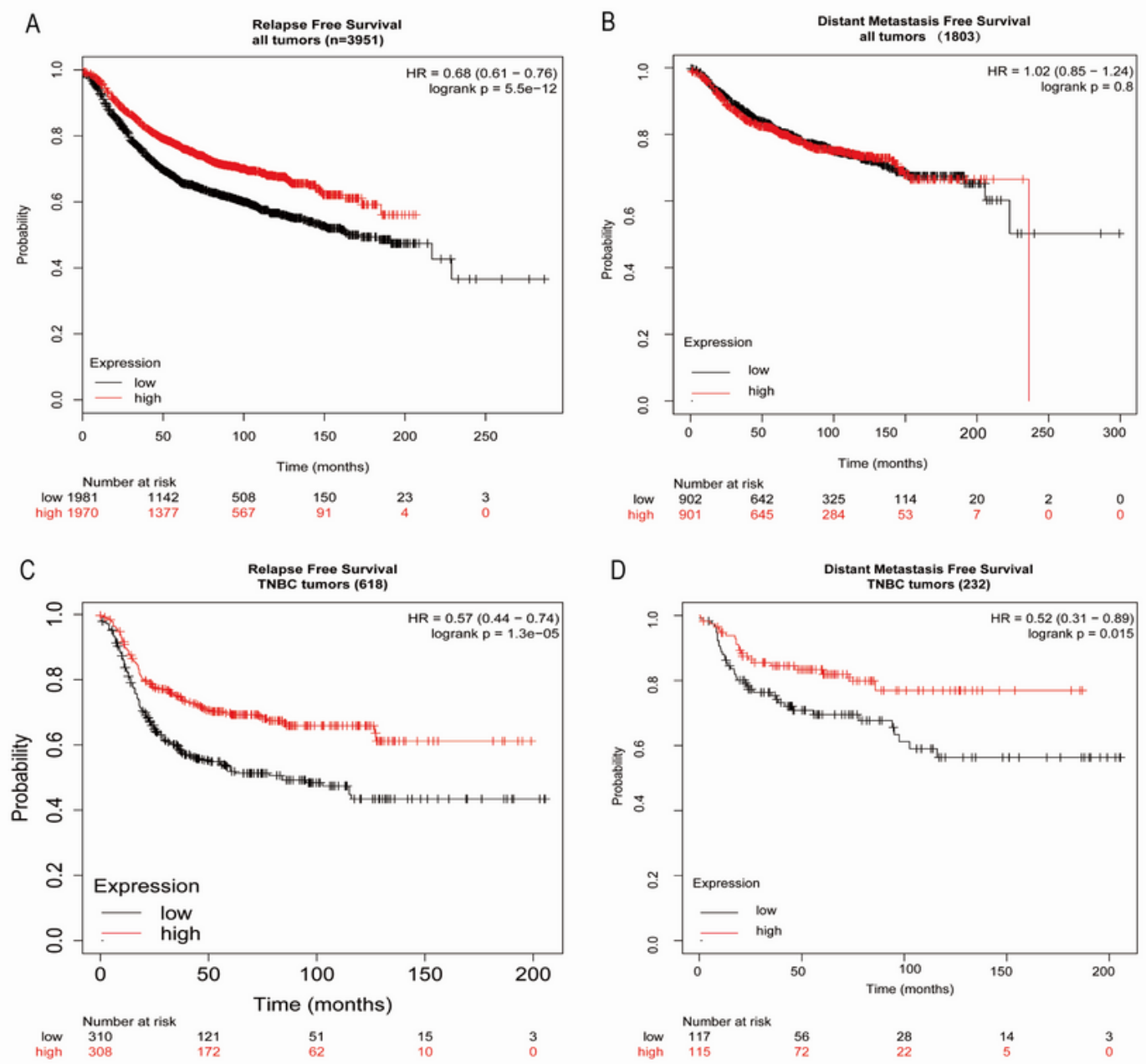

E

$\mathrm{F}$
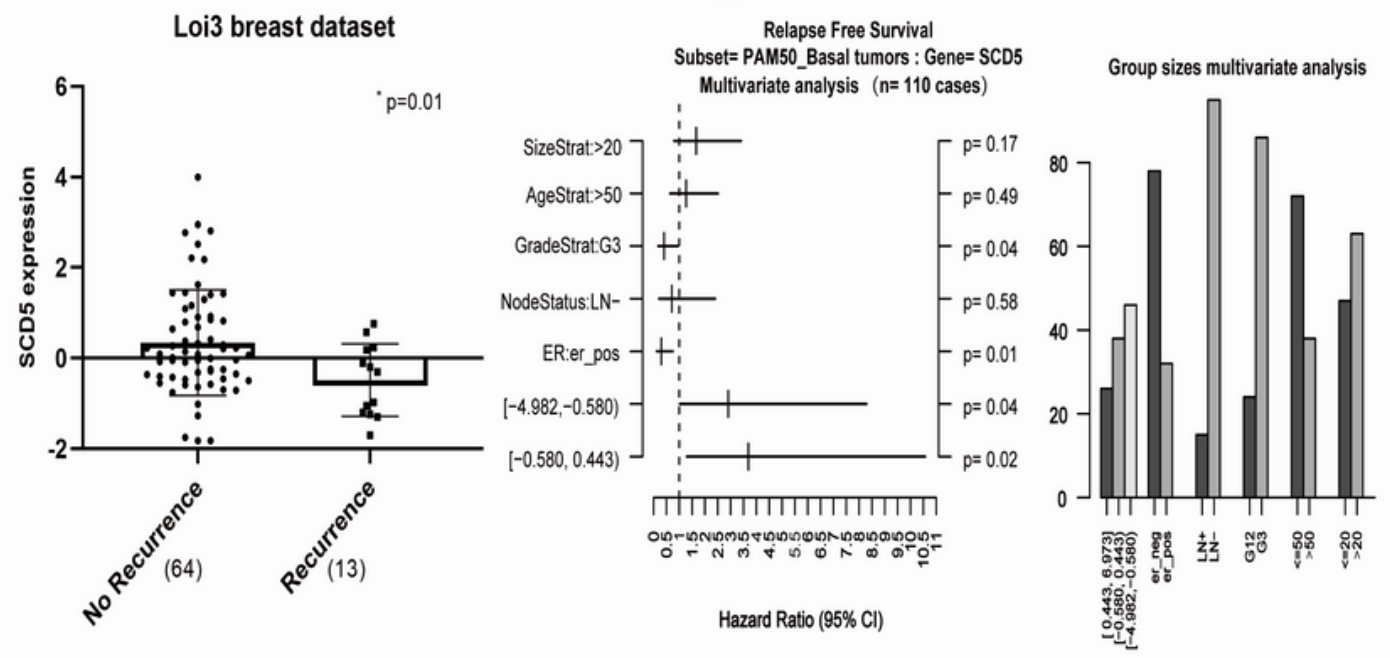

\section{Figure 3}

Survival analysis of SCD5 expression with RFS and DMFS in patients with breast cancer. (A-B). KaplanMeier relapse-free survival and distant metastasis-free survival curves of breast cancer patients (all molecular subtypes) with high or low SCD5 expression, using Kaplan-Meier plotter analysis. (C-D). Kaplan-Meier relapse-free survival and distant metastasis-free survival curves of TNBC patients with high or low SCD 5 expression, using Kaplan-Meier plotter analysis. (E). SCD5 expression in recurrence cohort 
and non-recurrence cohort using Loi3 breast dataset from Oncomine. (F). Multivariate Relapse-free survival analysis stratified by SCD 5 expression using dataset from GOBO.
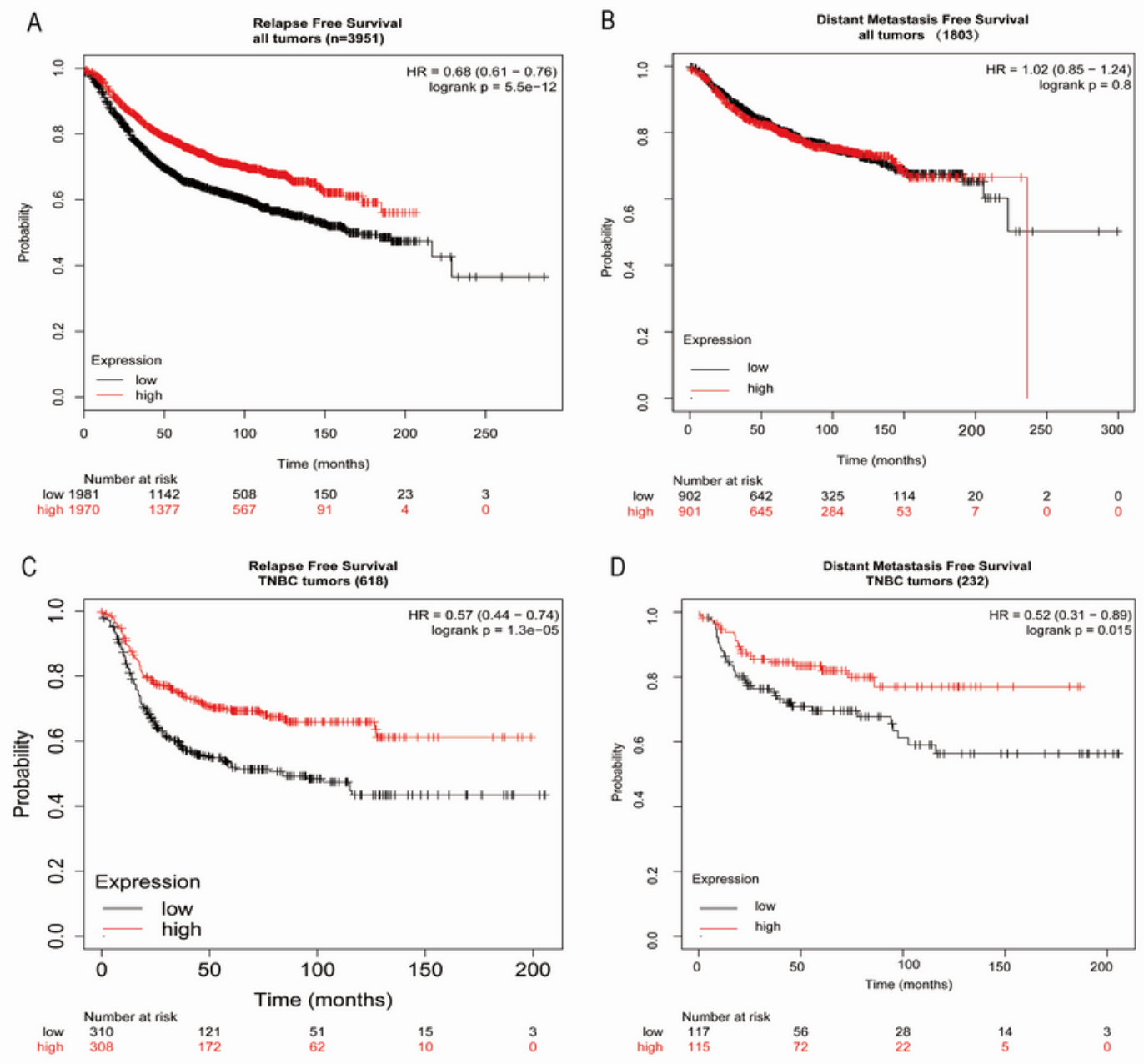

$E$

$\mathrm{F}$
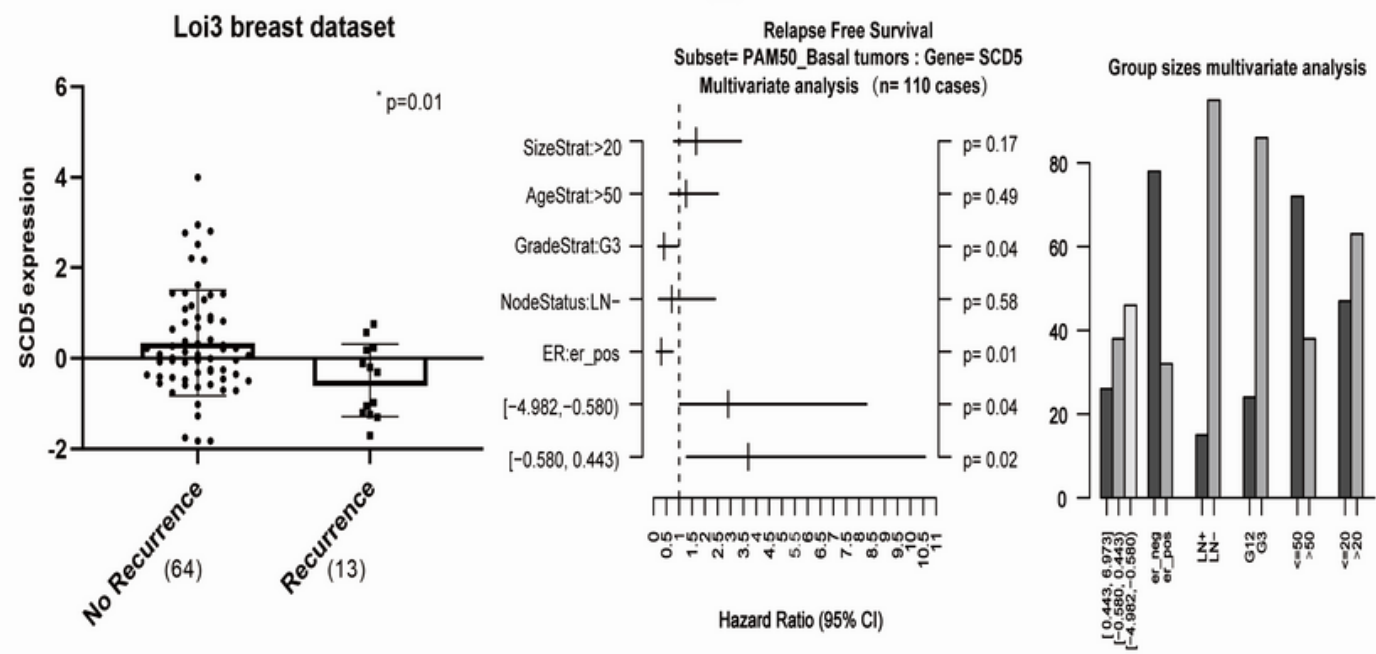

Figure 3

Survival analysis of SCD5 expression with RFS and DMFS in patients with breast cancer. (A-B). KaplanMeier relapse-free survival and distant metastasis-free survival curves of breast cancer patients (all molecular subtypes) with high or low SCD5 expression, using Kaplan-Meier plotter analysis. (C-D). 
Kaplan-Meier relapse-free survival and distant metastasis-free survival curves of TNBC patients with high or low SCD 5 expression, using Kaplan-Meier plotter analysis. (E). SCD5 expression in recurrence cohort and non-recurrence cohort using Loi3 breast dataset from Oncomine. (F). Multivariate Relapse-free survival analysis stratified by SCD 5 expression using dataset from GOBO.

A

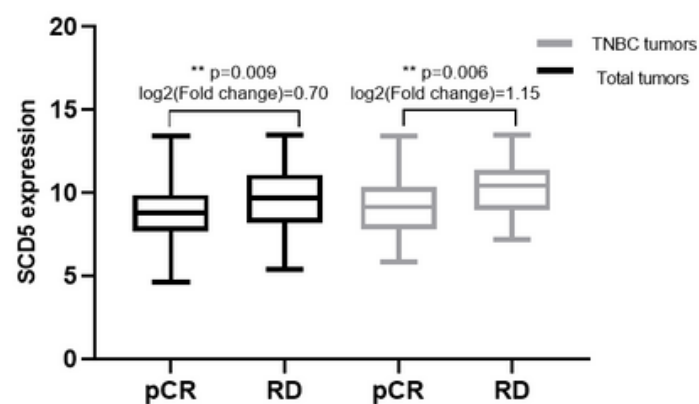

(56) (222)

(25) (46)

C

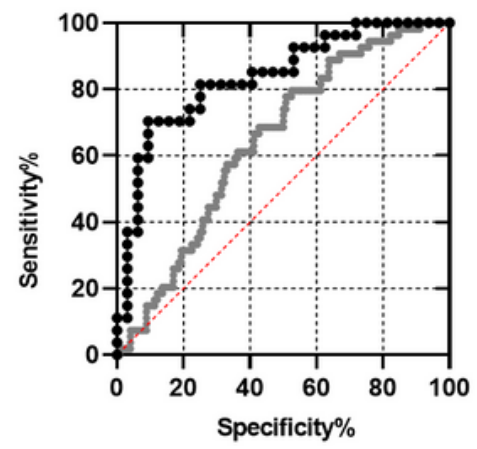

E

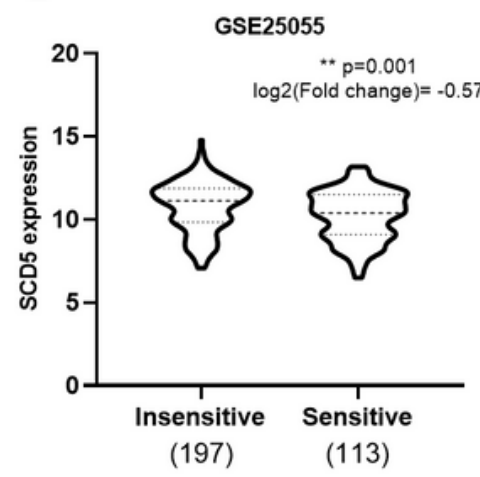

B

GSE20271

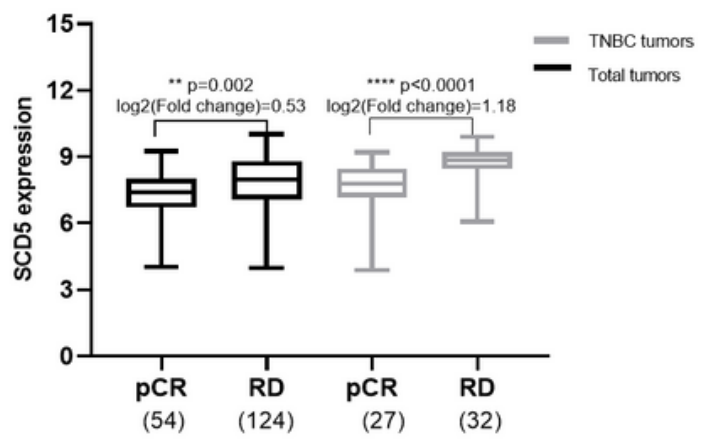

D

GSE20194

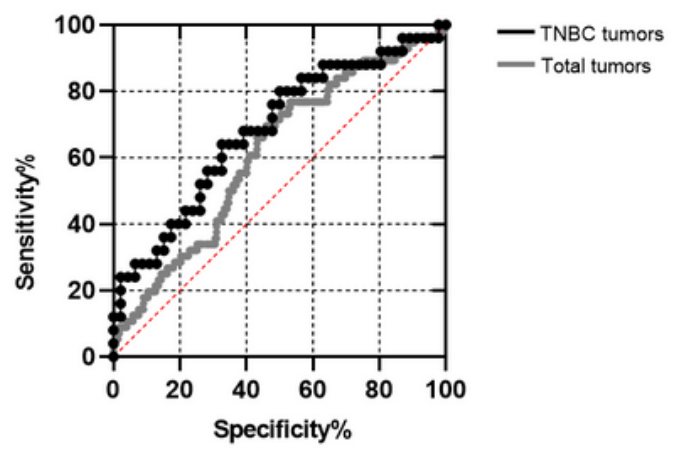

F

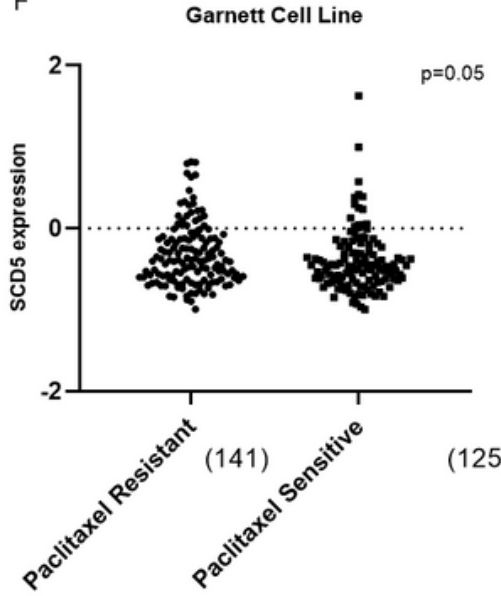

Figure 4 
Relationship between SCD5 expression and response to neoadjuvant chemotherapy. (A-B). SCD5 expression in $\mathrm{PCR}$ cohort (patients with pathological complete response to neoadjuvant chemotherapy) and RD cohort (patients with residual disease after neoadjuvant chemotherapy) using datasets from GEO. (C-D). ROC curves for SCD5 expression levels to discriminate the breast cancer patients (especially TNBC patients) who achieve pCR from non-pCR after neoadjuvant chemotherapy using data from GEO datasets. (E). SCD5 expression in anthracycline-taxane based neoadjuvant chemotherapy sensitive cohort and insensitive cohort using dataset from GEO. (F). SCD5 expression in paclitaxel-resistant cohort and paclitaxel-sensitive cohort using dataset from Oncomine. 
A

GSE20194

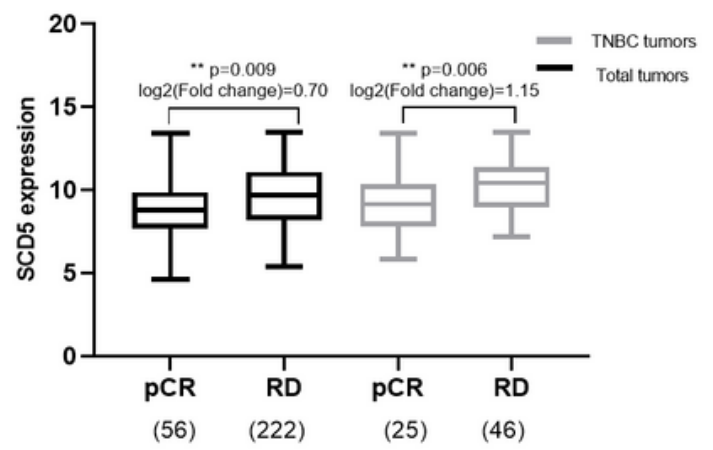

C

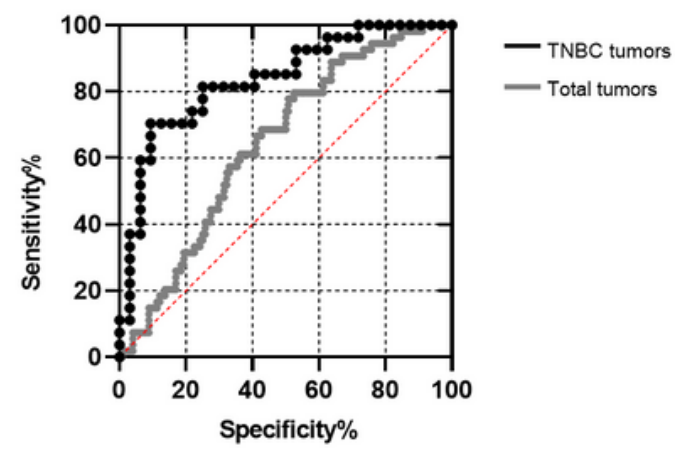

E

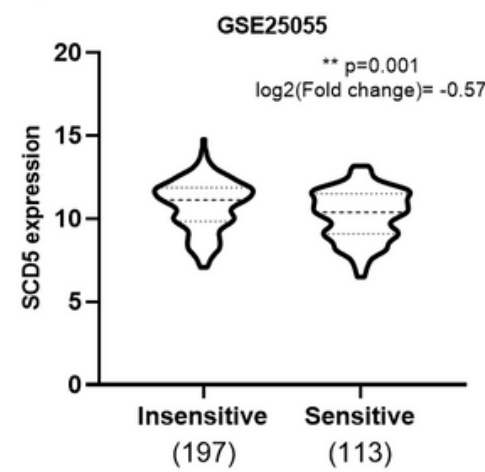

B

GSE20271

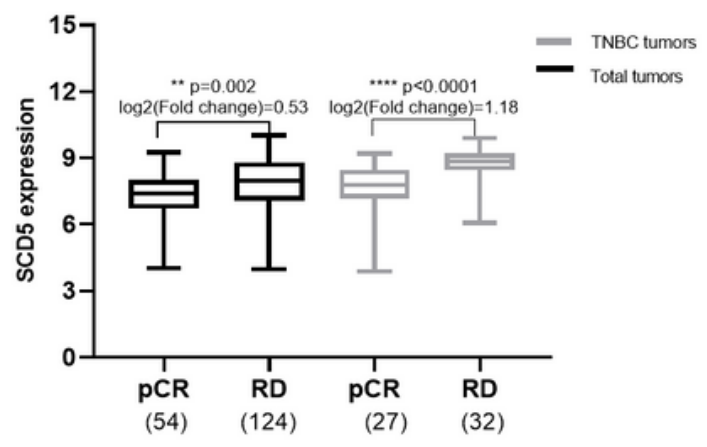

D

GSE20194

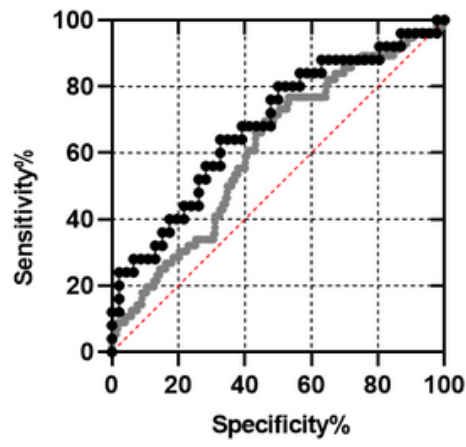

TNBC tumors
Total tumors

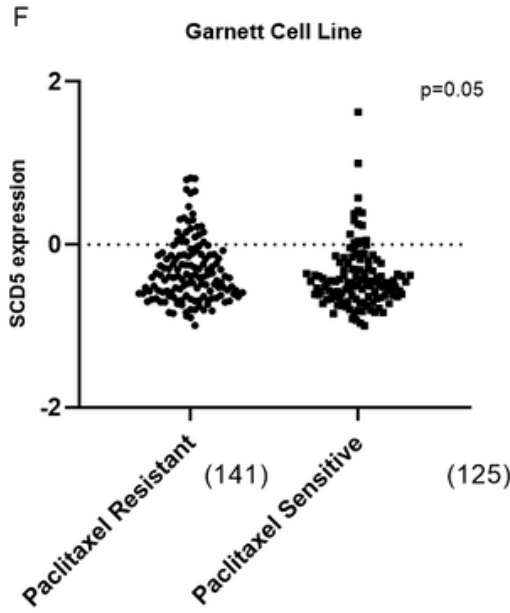

Figure 4

Relationship between SCD5 expression and response to neoadjuvant chemotherapy. (A-B). SCD5 expression in $\mathrm{pCR}$ cohort (patients with pathological complete response to neoadjuvant chemotherapy) and RD cohort (patients with residual disease after neoadjuvant chemotherapy) using datasets from GEO. (C-D). ROC curves for SCD5 expression levels to discriminate the breast cancer patients (especially TNBC patients) who achieve pCR from non-pCR after neoadjuvant chemotherapy using data from GEO 
datasets. (E). SCD5 expression in anthracycline-taxane based neoadjuvant chemotherapy sensitive cohort and insensitive cohort using dataset from GEO. (F). SCD5 expression in paclitaxel-resistant cohort and paclitaxel-sensitive cohort using dataset from Oncomine.

A

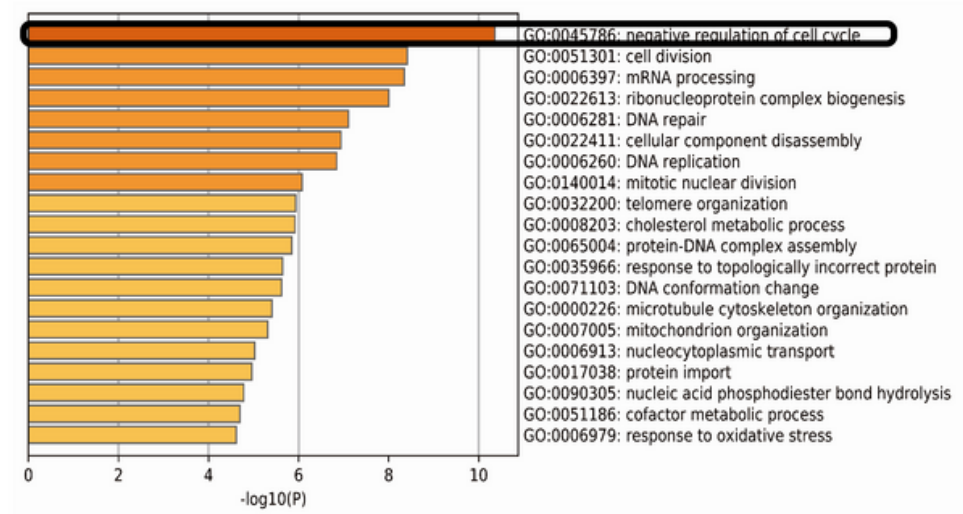

B

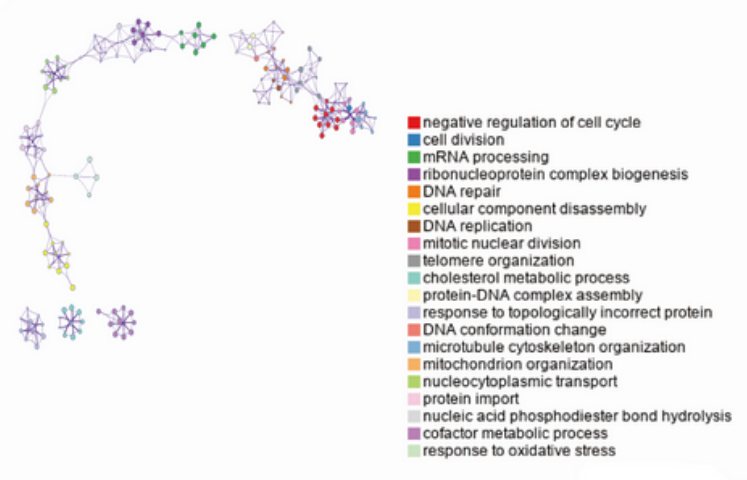

Figure 5

GO annotation and functional enrichment of SCD5-related differential expressed genes (DEGs). (A). GO biological process analysis of SCD5-related DEGs was performed by using Metascape with the criteria of $p$ value $<0.01$. (B). Network plot of the relationships among GO terms. Nodes represented enriched terms colored by its cluster identifier.

A

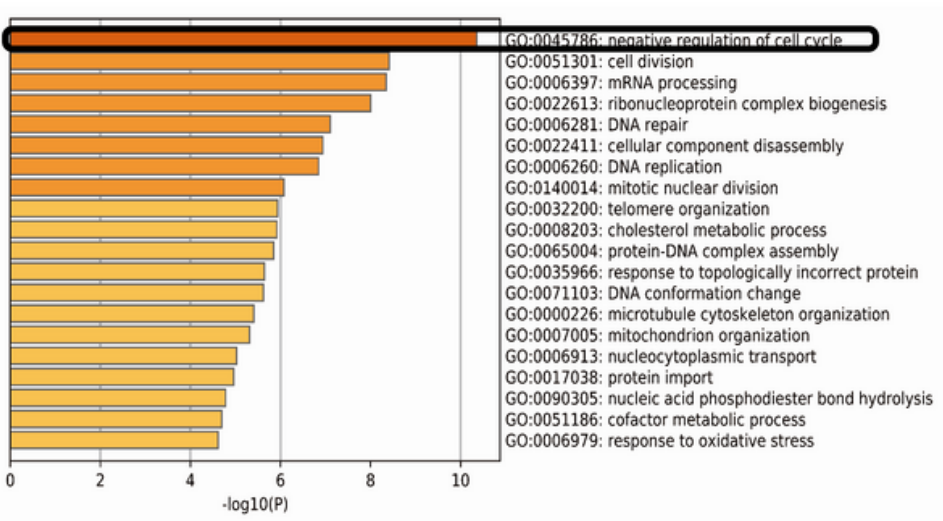

B

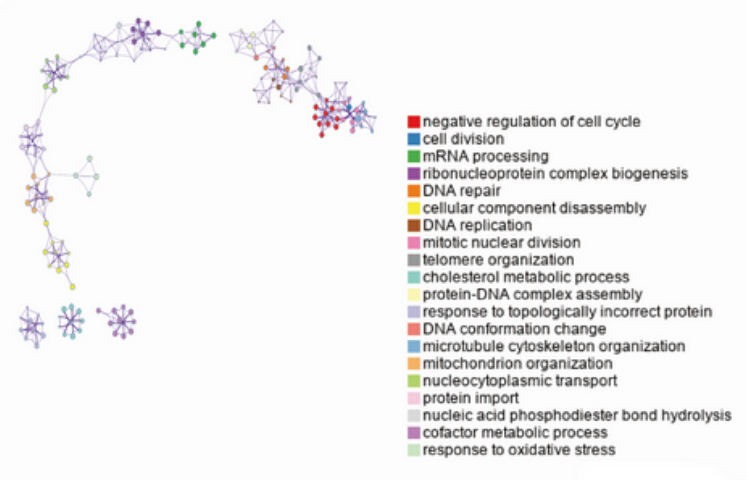

Figure 5

GO annotation and functional enrichment of SCD5-related differential expressed genes (DEGs). (A). GO biological process analysis of SCD5-related DEGs was performed by using Metascape with the criteria of $p$ value $<0.01$. (B). Network plot of the relationships among GO terms. Nodes represented enriched terms colored by its cluster identifier.

\section{Supplementary Files}


This is a list of supplementary files associated with this preprint. Click to download.

- TableS1.docx

- Tables1.docx

- Tables2.docx

- Tables2.docx

- FigureA1.tif

- FigureA1.tif

- FigureA2.tif

- FigureA2.tif

- FigureA3.tif

- FigureA3.tif

- FigureA4.tif

- FigureA4.tif

- supplementary.docx

- supplementary.docx 